Louisiana State University

LSU Digital Commons

$7-22-2013$

\title{
Mn1-xFexCoGe: A strongly correlated metal in the proximity of a noncollinear ferromagnetic state
}

\author{
Tapas Samanta \\ Southern Illinois University Carbondale \\ Igor Dubenko \\ Southern Illinois University Carbondale \\ Abdiel Quetz \\ Southern Illinois University Carbondale \\ Joseph Prestigiacomo \\ Louisiana State University \\ Philip W. Adams \\ Louisiana State University
}

See next page for additional authors

Follow this and additional works at: https://digitalcommons.Isu.edu/physics_astronomy_pubs

\section{Recommended Citation}

Samanta, T., Dubenko, I., Quetz, A., Prestigiacomo, J., Adams, P., Stadler, S., \& Ali, N. (2013).

Mn1-xFexCoGe: A strongly correlated metal in the proximity of a noncollinear ferromagnetic state.

Applied Physics Letters, 103 (4) https://doi.org/10.1063/1.4816381

This Article is brought to you for free and open access by the Department of Physics \& Astronomy at LSU Digital Commons. It has been accepted for inclusion in Faculty Publications by an authorized administrator of LSU Digital Commons. For more information, please contact ir@lsu.edu. 


\section{Authors}

Tapas Samanta, Igor Dubenko, Abdiel Quetz, Joseph Prestigiacomo, Philip W. Adams, Shane Stadler, and Naushad Ali 
Southern Illinois University Carbondale

OpenSIUC

Publications

Department of Physics

$7-2013$

\section{$\mathrm{Mn}_{1-\mathrm{x}} \mathrm{Fe}_{\mathrm{X}} \mathrm{CoGe}$ : A Strongly Correlated Metal in the Proximity of a Noncollinear Ferromagnetic State}

Tampas Samanta

Southern Illinois University Carbondale

Igor Dubenko

Southern Illinois University Carbondale

Abdiel Quetz

Southern Illinois University Carbondale

Joseph Prestigiacomo

Louisiana State University

Philip W. Adams

Louisiana State University

See next page for additional authors

Follow this and additional works at: http://opensiuc.lib.siu.edu/phys_pubs

(C) 2013 American Institute of Physics

Published in Applied Physics Letters, Vol.103 No. 042408 (2013) at doi: 10.1063/1.4816381

\section{Recommended Citation}

Samanta, Tampas, Dubenko, Igor, Quetz, Abdiel, Prestigiacomo, Joseph, Adams, Philip W., Stadler, Shane and Ali, Naushad. "Mn ${ }_{1-\mathrm{x}} \mathrm{Fe}_{\mathrm{x}} \mathrm{CoGe}$ A Strongly Correlated Metal in the Proximity of a Noncollinear Ferromagnetic State." (Jul 2013).

This Article is brought to you for free and open access by the Department of Physics at OpenSIUC. It has been accepted for inclusion in Publications by an authorized administrator of OpenSIUC. For more information, please contact opensiuc@lib.siu.edu. 
Authors

Tampas Samanta, Igor Dubenko, Abdiel Quetz, Joseph Prestigiacomo, Philip W. Adams, Shane Stadler, and Naushad Ali 


\title{
$\mathrm{Mn}_{1-\mathrm{x}} \mathrm{Fe}_{\mathrm{x}} \mathrm{CoGe}$ : A strongly correlated metal in the proximity of a noncollinear ferromagnetic state
}

\author{
Tapas Samanta, ${ }^{1}$ Igor Dubenko, ${ }^{1}$ Abdiel Quetz, ${ }^{1}$ Joseph Prestigiacomo, ${ }^{2}$ Philip W. Adams, ${ }^{2}$ \\ Shane Stadler, ${ }^{2}$ and Naushad Ali ${ }^{1}$ \\ ${ }^{1}$ Southern Illinois University, Carbondale, Illinois 62901, USA \\ ${ }^{2}$ Department of Physics \& Astronomy, Louisiana State University, Baton Rouge, Louisiana 70803, USA
}

(Received 28 May 2013; accepted 6 July 2013; published online 24 July 2013)

\begin{abstract}
An unusually large Kadowaki-Woods ratio of $\mathrm{A} / \gamma^{2} \sim 43 \mu \Omega \cdot \mathrm{cm} \cdot \mathrm{mol}^{2} \cdot \mathrm{K}^{2} \cdot \mathrm{J}^{-2}$ has been observed for intermetallic $\mathrm{Mn}_{1-\mathrm{x}} \mathrm{Fe}_{\mathrm{x}} \mathrm{CoGe}$ compounds in the proximity of $\mathrm{x}=0.2$ where the magnetic state of itinerant electrons system changes. The ratio is approximately four times larger than observed for heavy fermion systems. The manifestation of the strong electron correlations can be realized from the anisotropic origin of the effect through the substantial reduction of interlayer transport of heavy quasiparticles with comparable mean-free path and interlayer spacing in the proximity of a noncollinear ferromagnetic state associated with a large density of states at the Fermi level. (c) 2013 AIP Publishing LLC. [http://dx.doi.org/10.1063/1.4816381]
\end{abstract}

A central issue regarding the physics of strong electron correlations is the interplay between the itinerancy of electrons in solids due to wave function hybridization and localizing effects that are often rooted in electron-electron repulsion. ${ }^{1,2}$ As a result of these competing interactions, strongly correlated electron systems (SCES) exhibit a wide range of fascinating physical properties, such as heavy fermion behavior, metal-insulator transitions, high temperature superconductivity, colossal magnetoresistance, and so on. ${ }^{1,2}$ According to Fermi-liquid theory, for metallic systems the quadratic term of the resistivity, $\rho(\mathrm{T})=\rho_{0}+\mathrm{AT}^{2}$, is associated with electron-electron scattering at low temperature, and the electronic heat capacity varies linearly with temperature $\left(C_{\mathrm{el}}=\gamma \mathrm{T}\right)$. Therefore, the $\mathrm{A} / \gamma^{2}$, defined as the Kadowaki-Woods ratio (KWR), can be estimated from lowtemperature zero-field resistivity and heat capacity. The KWR is material specific, and can vary strongly with different classes of materials. Relatively small values of $\mathrm{A} / \gamma^{2} \sim 0.4 \mu \Omega \cdot \mathrm{cm} \cdot \mathrm{mol}^{2} \cdot \mathrm{K}^{2} \cdot \mathrm{J}^{-2}$ have been reported for transition metals. ${ }^{4}$ However, a large KWR has been observed in various types of SCES, such as heavy fermions (where $\mathrm{A} / \gamma^{2} \sim 10 \mu \Omega \cdot \mathrm{cm} \cdot \mathrm{mol}^{2} \cdot \mathrm{K}^{2} \cdot \mathrm{J}^{-2}$ ), ${ }^{3}$ transition metal oxides, ${ }^{5,6}$ organic charge-transfer salts, ${ }^{7,8}$ and has been recently observed in high $\mathrm{T}_{\mathrm{C}}$ superconductors based on FeAs. ${ }^{9}$ Several physical mechanisms have been proposed for explanation of the large KWR observed in the SCES. A unified model of KWR based on the dimensional analogy has been recently proposed in Ref. 10. It has been shown that the anisotropic transport of heavy quasiparticles (QP) in layered materials, such as transition-metal oxides and organic chargetransfer salts, can give rise to a large KWR. However, in the case of strongly correlated intermetallic compounds, the observation of a large KWR due to an anisotropic origin is rather uncommon. In this letter, we show that the large KWR observed in intermetallic $\mathrm{Mn}_{1-\mathrm{x}} \mathrm{Fe}_{\mathrm{x}} \mathrm{CoGe}$ compounds can be related to anisotropy of the strong electron correlations.

In recent years, considerable attention has been devoted to studies of MnCoGe-based systems due to temperature induced magneto-structural transitions resulting in shape memory phenomena, the giant magnetocaloric effects, and volume anomalies near room temperature. ${ }^{11,12}$ It is therefore of interest to conduct a deeper study of these systems perhaps to elucidate the physical origins of their pronounced physical properties. In particular, the rarely studied experimental electronic properties of MnCoGe-based systems may shed light on the behavior of this system. In fact, the major outcome of our experimental work suggests that $\mathrm{Mn}_{1}$ ${ }_{\mathrm{x}} \mathrm{Fe}_{\mathrm{x}} \mathrm{CoGe}$ in the vicinity of $\mathrm{x}=0.2$ can be considered as a strongly correlated metal.

According to neutron diffraction studies, stoichiometric $\mathrm{MnCoGe}$ and $\mathrm{FeCoGe}$ both have a collinear magnetic structure in the basal plane, and behave as an isotropic ferromagnet below their respective Curie temperatures of $\mathrm{T}_{\mathrm{C}} \sim 345$ and $370 \mathrm{~K} .{ }^{13} \mathrm{MnCoGe}$ has an orthorhombic TiNiSi-type structure in the ferromagnetically ordered state, but transforms to a hexagonal $\mathrm{Ni}_{2}$ In-type structure in the paramagnetic $(\mathrm{PM})$ state at about $\mathrm{T}_{\mathrm{M}} \sim 650 \mathrm{~K}$. However, FeCoGe crystallizes in a hexagonal phase which is stable in the entire temperature interval. Studies of isostructural MnNiGe-based systems indicate that an alternating sequence of $\mathrm{Ni}-\mathrm{Ge}$ and Mn layers perpendicular to the c-axis is formed in its hexagonal phase. ${ }^{14}$ A larger magnetic moment has been reported for the orthorhombic structure in comparison with that of the hexagonal phase, which is associated with the localized narrower $3 d$ band in the orthorhombic structure and a wider $3 d$ band in the hexagonal structure, resulting in itinerant character due to the decrease in bond length. ${ }^{15}$ Therefore, the substitution of $\mathrm{Fe}$ atoms for $\mathrm{Mn}$ in $\mathrm{Mn}_{1-\mathrm{x}} \mathrm{Fe}_{\mathrm{x}} \mathrm{CoGe}$ can stabilize the hexagonal structure at lower temperature with a reduced magnetic moment. It has been shown in Ref. 16 that, in the case of $\mathrm{CoMnX}(\mathrm{X}=\mathrm{P}, \mathrm{Si}, \mathrm{Ge})$, the collinear ferromagnetic (FM) phase with a high density of the electronic states is unstable compared to that in the noncollinear phase (antiferromagnetic or FM). A high number of states promotes a noncollinear magnetic arrangement in these types of compounds. ${ }^{16,17}$ The calculated total density of the states $\left(\mathrm{D}_{0}\left(\mathrm{E}_{\mathrm{F}}\right)\right)$ was found to be $\mathrm{D}_{0}\left(\mathrm{E}_{\mathrm{F}}\right)=5$ states/eV/f.u. for the parent MnCoGe. ${ }^{16}$ Therefore, a more than three-fold increase in $\mathrm{D}_{0}\left(\mathrm{E}_{\mathrm{F}}\right)$ as estimated for $\mathrm{Mn}_{0.8} \mathrm{Fe}_{0.2} \mathrm{CoGe}$ from the experimental results of the current work implies the 
existence of a noncollinear FM state in the hexagonal layered compound which results in the formation of massive QP. Our studies reveal an unusually large KWR of $\mathrm{A} / \gamma^{2} \sim 43 \mu \Omega \cdot \mathrm{cm} \cdot \mathrm{mol}^{2} \cdot \mathrm{K}^{2} \cdot \mathrm{J}^{-2}$. Therefore, strong electron correlations in the $\mathrm{Mn}_{1-\mathrm{x}} \mathrm{Fe}_{\mathrm{x}} \mathrm{CoGe}$ system can be associated with the anisotropic interlayer transport of heavy QP in the proximity of the changes in magnetic state of the itinerant electrons system induced by the increase in $\mathrm{Fe}$ concentration. The obtained results are explained in the context of a unified model of KWR.

Polycrystalline $\mathrm{Mn}_{1-\mathrm{x}} \mathrm{Fe}_{\mathrm{x}} \mathrm{CoGe}$ samples were prepared by arc-melting the constituent elements of purity better than 99.99\% in an ultra high purity argon atmosphere using water cooled massive bronze crucible and tungsten electrod, followed by annealing in high vacuum $\left(\approx 10^{-5}\right.$ Torr) for 4 days at $850^{\circ} \mathrm{C}$. The room temperature $\mathrm{X}$-ray diffraction patterns of the samples were obtained using $\mathrm{Cu} K \alpha$ radiation. Structural refinement was carried out using the Rietveld profile refinement method of the FULLPROF program. A superconducting quantum interference device magnetometer (SQUID, by Quantum Design, INC.) was employed to measure the magnetization of the samples within the temperature interval of $10-400 \mathrm{~K}$, and in applied magnetic fields up to $5 \mathrm{~T}$. The samples were heated to $380 \mathrm{~K}$ prior to magnetic measurements. The phase transitions temperatures, $\mathrm{T}_{\mathrm{C}}$ and $\mathrm{T}_{\mathrm{M}}$, were determined from the maximum of $\mathrm{dM} / \mathrm{dT}$ measured during heating in the presence of $1 \mathrm{kOe}$ magnetic field. The saturation magnetizations $\left(\mathrm{M}_{\mathrm{S}}\right)$ were obtained by extrapolating the high-field $\mathrm{M}(\mathrm{H})$ data to zero field at $\mathrm{T}=10 \mathrm{~K}$. The differential scanning calorimetry (DSC) measurements were carried out with a DSC 8000 (with a ramp rate of $20 \mathrm{~K} / \mathrm{min}$ during heating and cooling). Heat capacity $\left(\mathrm{C}_{\mathrm{P}}\right)$ measurements were done using a physical properties measurement system (PPMS by Quantum Design, INC.) using a vertical puck arrangement. Electrical resistivity $(\rho)$ measurements were performed using the standard four-probe method.

The X-ray diffraction patterns of $\mathrm{Mn}_{1-\mathrm{x}} \mathrm{Fe}_{\mathrm{x}} \mathrm{CoGe}$ as shown in Figs. 1(a)-1(c) confirm the hexagonal $\mathrm{Ni}_{2}$ In-type structure for $\mathrm{x}>0.09$ at room-temperature, except for small traces of the orthorhombic TiNiSi-type structure for $x=0.09$. The composition-dependent hexagonal lattice parameters a and $\mathrm{c}$ both decrease with increasing $\mathrm{Fe}$ concentration. According to the literature, with a smaller Mn-Mn separation, the MnCoGe-based system prefers to stabilize in the hexagonal phase. ${ }^{15,18}$ Therefore, the reduction of the Mn-Mn distance associated with the decrease of lattice parameters by the substitution of smaller $\mathrm{Fe}$ atoms for $\mathrm{Mn}$, stabilizes $\mathrm{Mn}_{1-\mathrm{x}} \mathrm{Fe}_{\mathrm{x}} \mathrm{CoGe}$ in the hexagonal $\mathrm{Ni}_{2} \mathrm{In}$-type structure. A linear decrease of $\mathrm{c}(\mathrm{x}) / \mathrm{a}(\mathrm{x})$ has been observed as shown in Fig. 1(d). However, the relative changes in $\mathrm{c}$ are more significant than those of a. For composition variations of $\mathrm{x}=0.09-0.4$, the lattice parameters decrease almost linearly from 4.095 to $4.080 \AA$ and from 5.296 to $5.208 \AA$ for a and c, respectively. Therefore, $\mathrm{c}$ decreases more rapidly compared to that of a, resulting in a decrease of c/a ratio associated with a smaller $\mathrm{Mn}-\mathrm{Mn} / \mathrm{Fe}$ separation along c-axis with increasing substitution of Fe. This indicates that Fe-substitution leads to a substantial reduction of the interlayer distance of $\mathrm{Mn} / \mathrm{Fe}$ atoms as separated by a $\mathrm{Co}-\mathrm{Ge}$ layer perpendicular to the c-axis. An alternating sequence of $\mathrm{Co}-\mathrm{Ge}$ and $\mathrm{Mn}$ layers in the $\mathrm{MnCoGe}-$ based systems are shown in the inset of Fig. 1(d). ${ }^{14,19}$

The variations of the transition temperatures $\left(T_{C}\right.$ and $\mathrm{T}_{\mathrm{M}}$ ) with $\mathrm{Fe}$ concentration, along with the temperature the peak positions of the DSC heating curves, are shown
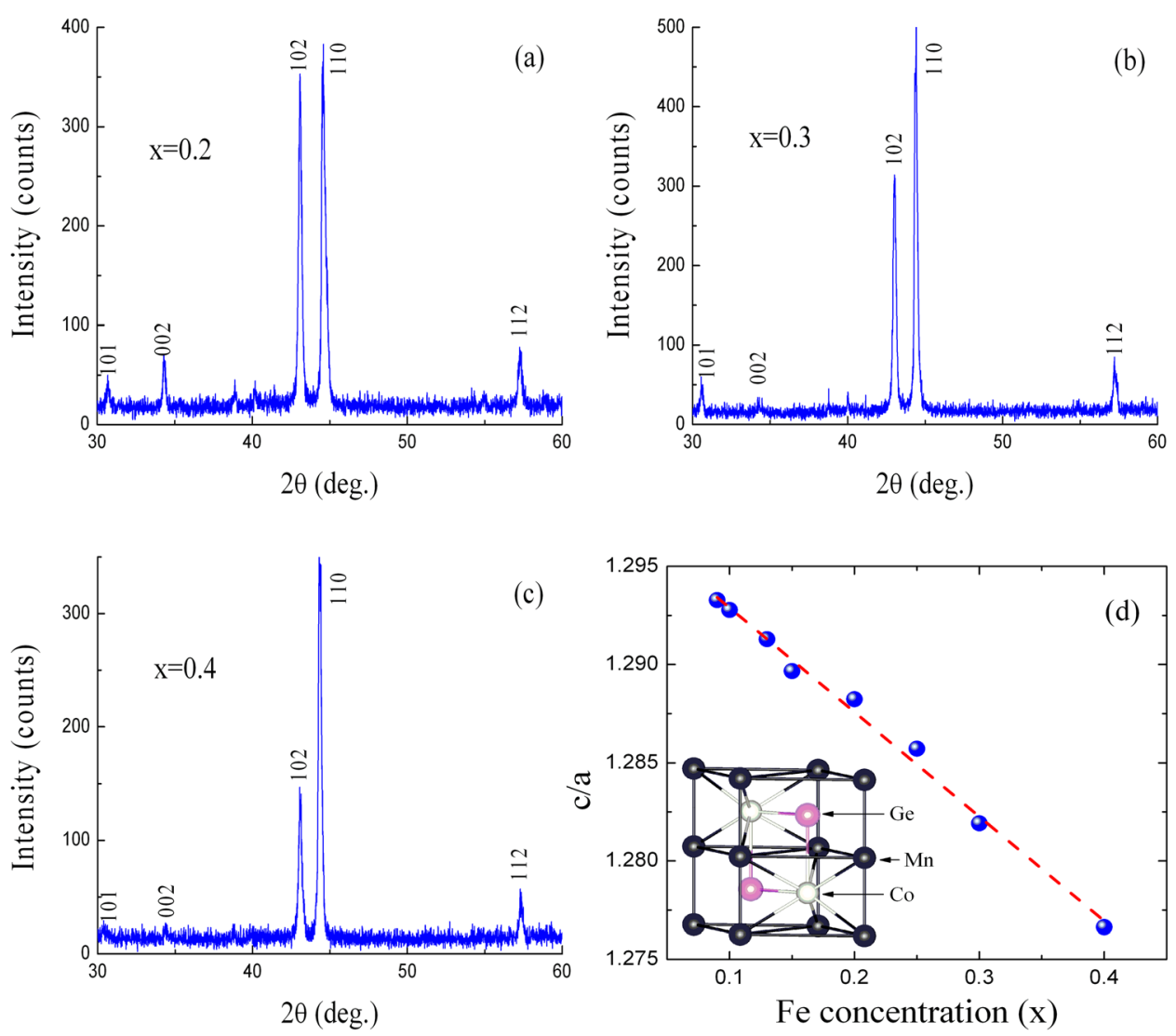

FIG. 1. Room temperature XRD patterns of $\mathrm{Mn}_{1-\mathrm{x}} \mathrm{Fe}_{\mathrm{x}} \mathrm{CoGe}$ for the compositions (a) $\mathrm{x}=0.2$, (b) $\mathrm{x}=0.3$, and (c) $\mathrm{x}=0.4$. (d) The ratio of hexagonal lattice parameters c/a at room temperature as a function of $\mathrm{Fe}$ concentration in $\mathrm{Mn}_{1-\mathrm{x}} \mathrm{Fe}_{\mathrm{x}} \mathrm{CoGe}$. The dashed line through the data points is a guide to the eye. Inset: an alternating sequence of Co-Ge and Mn layers as formed in hexagonal MnCoGe. The figure was adapted from Ref. 18. 


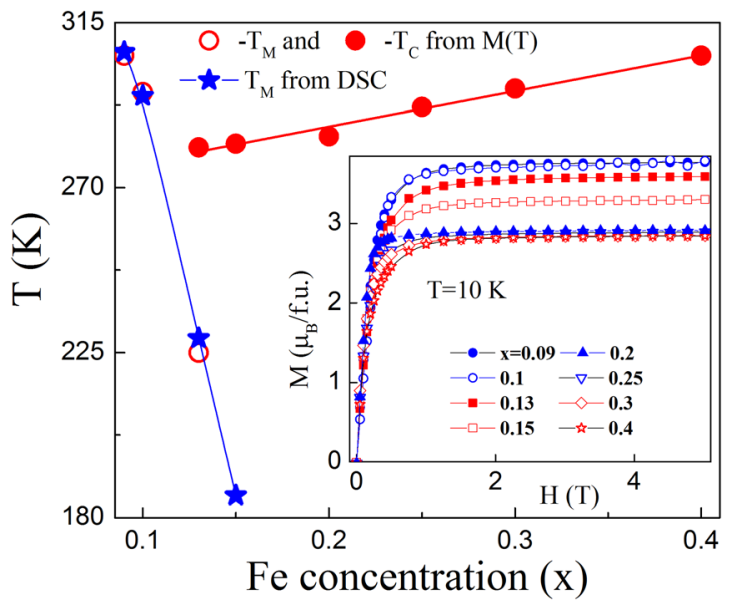

FIG. 2. The transition temperatures as a function of Fe concentration determined from magnetization $\mathrm{M}(\mathrm{T})$ and DSC data. Inset: Isothermal magnetization curves at $10 \mathrm{~K}$ for $\mathrm{Mn}_{1-\mathrm{x}} \mathrm{Fe}_{\mathrm{x}} \mathrm{CoGe}$.

in Fig. 2. The partial substitution of $\mathrm{Mn}$ by $\mathrm{Fe}$ in $\mathrm{Mn}_{1-\mathrm{x}} \mathrm{Fe}_{\mathrm{x}} \mathrm{CoGe}$ results in a coincidence of $\mathrm{T}_{\mathrm{C}}$ and $\mathrm{T}_{\mathrm{M}}$ for low Fe concentrations $(x=0.09$ and 0.1$)$, leading to a firstorder phase transition with a clearly visible thermal hysteresis between heating and cooling in both the M(T) and DSC curves. With a further increase of $\mathrm{x}$, a structurally driven first-order phase transition has been observed below $\mathrm{T}_{\mathrm{C}}$ up to $\mathrm{x}=0.15 .^{20}$ For $\mathrm{x} \geq 0.2$, a second-order magnetic transition, similar to that observed for the PM-FM transition with the hexagonal $\mathrm{Ni}_{2}$ In-type structure, was detected with no signature of a first-order transition. $\mathrm{T}_{\mathrm{M}}$ decreases sharply with increasing $\mathrm{x}$ and nearly vanishes for $\mathrm{x}=0.15$ with only small traces in the DSC curve. However, an increase of $\mathrm{T}_{\mathrm{C}}$ has been observed with increasing $x$. The $M(H)$ curves show a shape typical for FM type ordering (see inset of Fig. 2). The saturation magnetizations $\left(\mathrm{M}_{\mathrm{S}}\right)$ initially decrease with increasing $\mathrm{x}$ but remain nearly constant (about $2.8 \mu_{\mathrm{B}} /$ f.u.) in the hexagonal phase for $x \geq 0.2$ ((see Fig. 3(b)).

Representative data of the temperature-dependent inverse susceptibility $\left(\chi^{-1}\right)$ in the presence of $1 \mathrm{kOe}$ for $\mathrm{x}=0.1$ and 0.2 are plotted in Fig. 3(a). In the PM state, the linear variations of $\chi^{-1}(\mathrm{~T})$ for small $\mathrm{x}$ were well-fitted by the standard Curie-Weiss term, indicating a localized moment behavior. However, a modified Curie-Weiss expression, $\chi=\chi_{0}+\mathrm{C} /(\mathrm{T}$ $\theta_{\mathrm{P}}$ ) (where $\chi_{0}=\mathrm{T}$-independent susceptibility, $\mathrm{C}=$ Curie constant, and $\theta_{\mathrm{P}}=\mathrm{PM}$ Curie temperature) was used to fit the nonlinear $\chi^{-1}(\mathrm{~T})$ curve for higher Fe concentrations, suggesting a temperature-independent contribution from itinerant behavior. From the fitting, the values of $\mathrm{C}$ and $\theta_{\mathrm{P}}$ were obtained for different $x$. The effective magnetic moment $\left(\mu_{\text {eff }}\right)$ was determined from the Curie constant C. According RhodesWohlfarth model, a value of $\mathrm{P}_{\mathrm{C}} / \mathrm{M}_{\mathrm{S}}$ (where $\mathrm{P}_{\mathrm{C}}$ is total spin angular moment and $\left.\mathrm{P}_{\mathrm{C}}\left(\mathrm{P}_{\mathrm{C}}+2\right) \equiv \mu_{\mathrm{eff}}{ }^{2}\right)$ greater than the unity implies an itinerant moment system. ${ }^{21}$ Therefore, a large $\mathrm{P}_{\mathrm{C}}$ $\mathrm{M}_{\mathrm{S}} \sim 2.2 \mu_{\mathrm{B}} /$ f.u. for $\mathrm{x} \geq 0.2$ (see Fig. 3(b)) indicates a significant contribution due to itinerant magnetic behavior of the $\mathrm{Mn}_{1-\mathrm{x}} \mathrm{Fe}_{\mathrm{x}} \mathrm{CoGe}$ compounds. A hydrostratic pressure study on the $\mathrm{MnCoGe-based} \mathrm{system} \mathrm{also} \mathrm{indicated} \mathrm{an} \mathrm{itinerant} \mathrm{charac-}$ ter of the 3d-subsystem in these types of compounds. ${ }^{22}$ Thus, $\mathrm{Fe}$ in $\mathrm{Mn}_{1-\mathrm{x}} \mathrm{Fe}_{\mathrm{x}} \mathrm{CoGe}$ intermetallics generally contributes to the itinerant component of the $3 \mathrm{~d}$-subsystem.

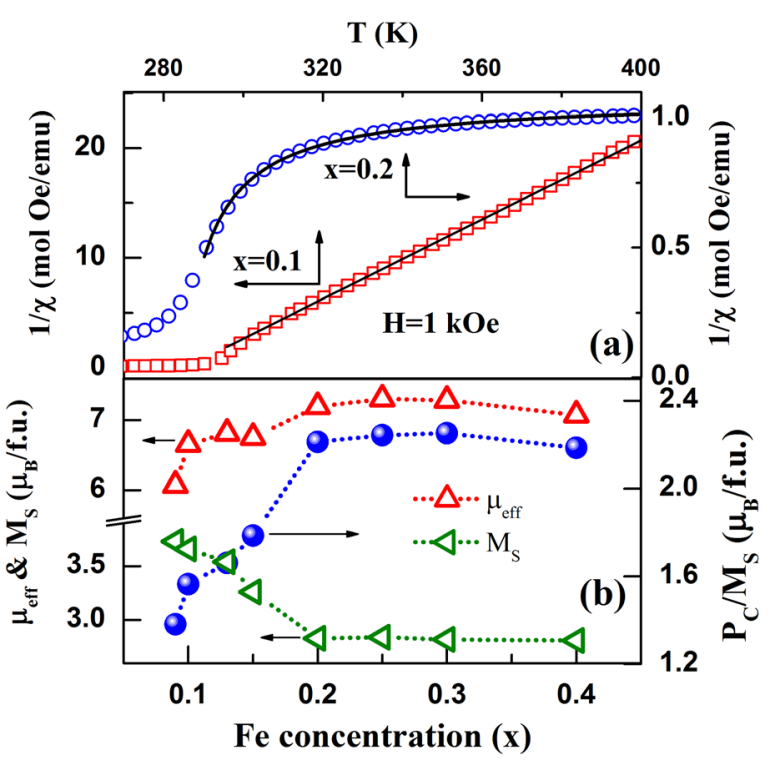

FIG. 3. (a) Inverse susceptibility $\left(\chi^{-1}\right)$ versus temperature $(\mathrm{T})$ in the presence of $1 \mathrm{kOe}$ field, and the corresponding fitting as indicated by the solid curves for $\mathrm{x}=0.1$ and 0.2 , respectively. (b) Concentration dependencies of the effective magnetic moment $\left(\mu_{\mathrm{eff}}\right)$, saturation magnetization $\left(\mathrm{M}_{\mathrm{S}}\right)$, and $\mathrm{P}_{\mathrm{C}} / \mathrm{M}_{\mathrm{S}}$.

The development of strong electron correlations in this system arises when it passes through the noncollinear FM state for $\mathrm{x}=0.2$, as evidenced from our $\mathrm{C}_{\mathrm{P}}(\mathrm{T})$ and $\rho(\mathrm{T})$ data. The zero-field $\mathrm{C}_{\mathrm{P}}(\mathrm{T})$ data for $\mathrm{x}=0.2$ is shown in Fig. 4(a). A small peak has been detected at $\mathrm{T}_{\mathrm{C}}$. The low temperature data shown in the inset of Fig. 4(a) was fitted by the standard expression, $\mathrm{C}_{\mathrm{P}}=\gamma \mathrm{T}+\beta \mathrm{T}^{3}$, and the fitted values of $\gamma$ and $\beta$ were found to be $27.6 \mathrm{~mJ} / \mathrm{mol} \mathrm{K}^{2}$ and $0.164 \mathrm{~mJ} / \mathrm{mol} \mathrm{K}^{4}$, respectively. The large value of $\gamma$ indicates the formation of heavy QP with a mass enhancement of approximately 30 times that of free electrons. The Debye temperature obtained

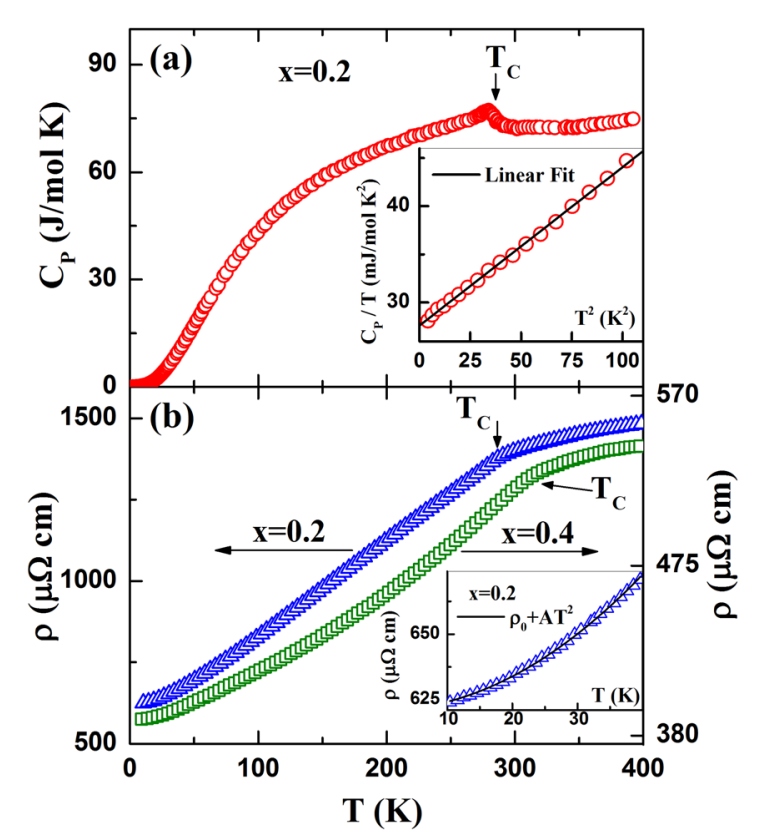

FIG. 4. (a) Heat capacity $\left(C_{P}\right)$ versus temperature (T) for $x=0.2$. Inset: the linear fit of $\mathrm{C}_{\mathrm{P}} / \mathrm{T}$ versus $\mathrm{T}^{2}$. (b) Electrical resistivity $(\rho)$ as a function of $\mathrm{T}$ with $\mathrm{x}=0.2$ and 0.4 . Inset: the fit of $\rho(\mathrm{T})$ by $\rho(\mathrm{T})=\rho_{0}+\mathrm{AT}^{2}$ for $\mathrm{x}=0.2$ as indicated by the solid curve. 
from the $\beta$ value is $306 \mathrm{~K}$. The DOS at $\mathrm{E}_{\mathrm{F}}$ estimated from the $\gamma$ value is $\mathrm{D}_{0}\left(\mathrm{E}_{\mathrm{F}}\right)=16.5$ states/eV/f.u. Previously it has been shown that, in the case of the CoMnX $(X=P, S i, G e)$, the noncollinear ground states (antiferromagnetic or ferromagnetic) are energetically preferable compared to the collinear FM phase as characterized by the low total density of states at the Fermi level, $D_{0}\left(E_{F}\right)$. A high number of states can make the collinear FM state unstable and promotes a noncollinear magnetic arrangement in these types of compounds, ${ }^{16,17}$ Therefore, a more than three-fold increase in $\mathrm{D}_{0}\left(\mathrm{E}_{\mathrm{F}}\right)$ for $\mathrm{Mn}_{0.8} \mathrm{Fe}_{0.2} \mathrm{CoGe}$ relative to that of the parent $\mathrm{MnCoGe}$ $\left(\mathrm{D}_{0}\left(\mathrm{E}_{\mathrm{F}}\right)=5 \text { states/eV/f.u. }\right)^{16}$ suggests the instability of the collinear FM state in this composition and promotes a stable noncollinear FM state. A noncollinear magnetic state can be stabilized in any elements, alloys, or compounds through the formation of a hybridization gap at the Fermi energy, as emphasized previously, ${ }^{23}$ and was recently found the related system $\mathrm{CoMnSi}{ }^{16,17}$

The temperature dependence of the resistivity $(\rho)$ in the absence of magnetic field is shown in Fig. 4(b) for the compounds with $\mathrm{x}=0.2$ and 0.4 . The $\rho(\mathrm{T})$ data suggest metallic ground states for both compositions. A rather large residual resistivity for $\mathrm{x}=0.2\left(\rho_{0}=624 \mu \Omega \mathrm{cm}\right)$ compared to most conventional metallic conductors seems to be related to interlayer transport associated with a substantial reduction of the meanfree path of heavy quasiparticles. As mentioned previously, the compositions for $\mathrm{x} \geq 0.2$ have a stable hexagonal $\mathrm{Ni}_{2} \mathrm{In}$-type structure without a first-order structural transition. An alternating sequence of Co-Ge and $\mathrm{Mn}$ layers perpendicular to the caxis is formed in hexagonal structure of $\mathrm{MnCoGe}$ as reported earlier on isostructural hexagonal MnNiGe-based systems. ${ }^{14} \mathrm{~A}$ change of slope has been observed in $\rho(\mathrm{T})$ in the vicinity of $\mathrm{T}_{\mathrm{C}}$. The low-temperature $\rho(\mathrm{T})$ data for $\mathrm{x}=0.2$ was fitted by $\rho(\mathrm{T})=\rho_{0}+\mathrm{AT}^{2}$, suggesting a dominant electron-electron scattering behavior (see the inset of Fig. 4(b)). The value of A obtained from the fit is $0.0324 \pm 0.0002 \mu \Omega \cdot \mathrm{cm} \cdot \mathrm{K}^{-2}$. Therefore, the Kadowaki-Woods ratio for $\mathrm{x}=0.2$ is $\mathrm{KWR}=\mathrm{A} / \gamma^{2}=43 \mu \Omega \cdot \mathrm{cm} \cdot \mathrm{mol}^{2} \cdot \mathrm{K}^{2} \cdot \mathrm{J}^{-2}$. This value is much larger than the value $\mathrm{A} / \gamma^{2} \sim 10 \mu \Omega \cdot \mathrm{cm} \cdot \mathrm{mol}^{2} \cdot \mathrm{K}^{2} \cdot \mathrm{J}^{-2}$ reported for a number of heavy fermion compounds, ${ }^{3}$ suggesting that $\mathrm{Mn}_{0.8} \mathrm{Fe}_{0.2} \mathrm{CoGe}$ is a strongly correlated metal. Although the studied materials were synthesized from isotropic endmembers (MnCoGe and $\mathrm{FeCoGe}$ ) similar to that of heavy fermion compounds, the development of strong electron correlation in $\mathrm{Mn}_{0.8} \mathrm{Fe}_{0.2} \mathrm{CoGe}$ has an unexpected and unique anisotropic origin. In this case, interlayer transport of heavy QP due to the formation of a layered structure is the origin of the strong electron correlation. This will be explained in the context of the unified model developed recently by Jacko et al. ${ }^{10}$ to describe the KWR by considering a dimensional analysis.

The model by Jacko et al. employs a normalized KWR in the form of $\mathrm{Af}_{\mathrm{dx}}(\mathrm{n}) / \gamma^{2}=81 / 4 \pi \hbar \mathrm{k}_{\mathrm{B}}^{2} \mathrm{e}^{2}$, according to which a broad class of strongly correlated metals takes a single value. In this $\mathrm{KWR}, \mathrm{f}_{\mathrm{dx}}(\mathrm{n})$ represents the dimensional term $\left(\mathrm{d}=\right.$ dimensionality) with $\mathrm{f}_{\mathrm{dx}}(\mathrm{n}) \equiv \mathrm{nD}_{0}{ }^{2}\left\langle\nu_{0 \mathrm{x}}{ }^{2}\right\rangle \xi^{2}$, where $\mathrm{n}, \mathrm{D}_{0}$, and $\left\langle\nu_{0 \mathrm{x}}{ }^{2}\right\rangle$ represent the conduction-electron density, the DOS at $\mathrm{E}_{\mathrm{F}}$, and the Fermi velocity, respectively, and $\xi \approx 1$ is a pure number. For anisotropic layered materials, $\left\langle\nu_{0 \mathrm{x}}{ }^{2}\right\rangle$ may vary in the different crystallographic directions. In this model, they found for quasi-2D layered materials that $\mathrm{f}_{2 \|}(\mathrm{n})=\mathrm{n}^{2} / \pi \mathrm{c} \hbar^{2}$ (c = interlayer spacing) and $\mathrm{f}_{2 \perp}(\mathrm{n})=2 \mathrm{~nm}_{0}{ }^{2} \mathrm{t}_{\perp 0}{ }^{2} / \pi^{2} \hbar^{6}$. Notably, the interlayer hopping integral $\left(t_{\perp 0}\right)$ is the most important parameter for layered systems, whether it is via coherent transport or thermally assisted hopping. The above-described formalism fits quite well for reported layer-type SCES, which exhibit large values of $\mathrm{A} / \gamma^{2}$ without considering $f_{d x}(n)$, and becomes single-valued after the addition of $f_{d x}(n)$. In other words, the relatively small value of $f_{d x}(n)$ is solely responsible for the large $\mathrm{A} / \gamma^{2}$ observed in layered SCES. Therefore, the substantial reduction of $t_{\perp 0}$ due to the decrease of interlayer QP transport makes $f_{2 \perp}(n)$ (i.e., effectively $f_{d x}(n)$ ) small, and enhances the $A / \gamma^{2}$ value for layered SCES. At $300 \mathrm{~K}$, the measured $\rho$ are 1399 and $518 \mu \Omega \mathrm{cm}$ for $\mathrm{x}=0.2$ and 0.4 , respectively. These correspond to a normalized mean-free path of $\mathrm{k}_{\mathrm{F}} \ell \approx \mathrm{h}(\mathrm{c} / 2) / \mathrm{e}^{2} \rho \approx 0.5$ and 1.3 along the direction perpendicular to the atomic layers with $\mathrm{c}=5.28$ and $5.22 \AA$ for $\mathrm{x}=0.2$ and 0.4 , respectively (where $\mathrm{c}=$ lattice constant along $\mathrm{c}$-axis, $\mathrm{c} / 2=$ interlayer spacing of atomic planes of $\mathrm{Mn}$ or $\mathrm{Mn}-\mathrm{Fe}$, and $\mathrm{h} / \mathrm{e}^{2} \approx 26 \mathrm{k} \Omega$ is the quantum resistance), which suggest a higher degree of metallicity for the composition with $\mathrm{x}=0.4$ compared to that of $\mathrm{x}=0.2$. The substantial reduction of $\mathrm{k}_{\mathrm{F}} \ell$ makes it comparable with $\mathrm{c} /$ 2 in the proximity of the noncollinear FM state for $\mathrm{x}=0.2$. As a result, the interlayer QP transport $\left(t_{\perp 0}\right)$ at low temperature decreases markedly, which ensures that it becomes a SCES with large $\mathrm{A} / \gamma^{2}$ for $\mathrm{x}=0.2$ due to the substantial reduction of the effective $f_{d x}(n)$. Moreover, it would be reasonable to anticipate that a large $\mathrm{D}_{0}\left(\mathrm{E}_{\mathrm{F}}\right)$ should result in a narrower effective bandwidth $\left(\mathrm{W}=4 \mathrm{t}_{\perp 0}\right)$ for $\mathrm{x}=0.2$, and therefore $t_{\perp 0}$ should be reduced. The system moves towards a collinear FM state having normal 3D metallic behavior (as observed for $\mathrm{x}=0.4$ with large normalized mean-free path perpendicular to the atomic layers in comparison to $\mathrm{x}=0.2$ ) with $x>0.2$ after exhibiting a noncollinear FM state for $\mathrm{x}=0.2$. The trend of the normal metallic behavior for higher Fe concentrations is well supported by the collinear behavior reported for FeCoGe. Transport and neutron diffraction studies on a single crystal sample would be beneficial to understand the unusual type of strong electron correlation in this system, and will be a topic of future studies.

As explored in this study, a noncollinear stability in a FM material can lead to strong electron correlations. The substantial reduction of interlayer QP transport with a comparable mean-free path and interlayer spacing in the proximity of noncollinear FM state results in a large $\mathrm{A} / \gamma^{2} \sim 43 \mu \Omega \cdot \mathrm{cm} \cdot \mathrm{mol}^{2} \cdot \mathrm{K}^{2} \cdot \mathrm{J}^{-2}$ in $\mathrm{Mn}_{1-\mathrm{x}} \mathrm{Fe}_{\mathrm{x}} \mathrm{CoGe}$ as consequence of strong electron correlations.

This work was supported by the Office of Basic Energy Sciences, Material Science Division of the U.S. Department of Energy (Grant Nos. DE-FG02-06ER46291 and DE-FG0213ER46946). P.W.A. acknowledges the support of the U.S. DOE under Grant No. DE-FG02-07ER46420.

\footnotetext{
${ }^{1}$ M. Imada, A. Fujimori, and Y. Tokura, Rev. Mod. Phys. 70, 1039 (1998). ${ }^{2}$ D. N. Basov, R. D. Averitt, D. v. d. Marel, M. Dressel, and K. Haule, Rev. Mod. Phys. 83, 471 (2011).

${ }^{3}$ K. Kadowaki and S. B. Woods, Solid State Commun. 58, 507 (1986).

${ }^{4}$ M. J. Rice, Phys. Rev. Lett. 20, 1439 (1968).

${ }^{5}$ N. E. Hussey, J. Phys. Soc. Jpn. 74, 1107 (2005).
} 
${ }^{6}$ S. Y. Li, L. Taillefer, D. G. Hawthorn, M. A. Tanatar, J. Paglione, M. Sutherland, R. W. Hill, C. H. Wang, and X. H. Chen, Phys. Rev. Lett. 93, 056401 (2004).

${ }^{7}$ M. Dressel, G. Grüner, J. E. Eldridge, and J. M. Williams, Synth. Met. 85, 1503 (1997).

${ }^{8}$ B. J. Powell and R. H. McKenzie, J. Phys.: Condens. Matter 18, R827 (2006).

${ }^{9}$ A. Pandey, R. S. Dhaka, J. Lamsal, Y. Lee, V. K. Anand, A. Kreyssig, T. W. Heitmann, R. J. McQueeney, A. I. Goldman, B. N. Harmon, A. Kaminski, and D. C. Johnston, Phys. Rev. Lett. 108, 087005 (2012).

${ }^{10}$ A. C. Jacko, J. O. Fjaerestad, and B. J. Powell, Nature Phys. 5, 422 (2009).

${ }^{11}$ T. Samanta, I. Dubenko, A. Quetz, S. Stadler, and N. Ali, Appl. Phys. Lett. 101, 242405 (2012).

${ }^{12}$ N. T. Trung, L. Zhang, L. Caron, K. H. J. Buschow, and E. Brück, Appl. Phys. Lett. 96, 172504 (2010).

${ }^{13}$ A. SzytuŁa, A. T. Pedziwiatr, Z. Tomkowicz, and W. Bażela, J. Magn. Magn. Mater. 25, 176 (1981).

${ }^{14}$ E. Liu, W. Wang, L. Feng, W. Zhu, G. Li, J. Chen, H. Zhang, G. Wu, C. Jiang, H. Xu, and F. d. Boer, Nature Commun. 3, 873 (2012).
${ }^{15}$ J. T. Wang, D. S. Wang, C. Chen, O. Nashima, T. Kanomata, H. Mizuseki, and Y. Kawazoe, Appl. Phys. Lett. 89, 262504 (2006).

${ }^{16}$ A. Barcza, Z. Gercsi, K. S. Knight, and K. G. Sandeman, Phys. Rev. Lett. 104, 247202 (2010).

${ }^{17}$ Z. Gercsi, K. Hono, and K. G. Sandeman, Phys. Rev. B 83, 174403 (2011).

${ }^{18}$ W. Zhang, O. Tegus, Y. Wu, Yirgeltu, H. Yan, and S. Lin, J. Mater. Sci. Technol. 25, 781 (2009).

${ }^{19}$ P. E. Markin, N. V. Mushnikov, V. I. Khrabrov, and M. A. Korotin, Phys. Met. Metallogr. 106, 481 (2008).

${ }^{20}$ L. Caron, N. T. Trung, and E. Brück, Phys. Rev. B 84, 020414(R) (2011).

${ }^{21}$ P. R. Rhodes and E. P. Wohlfarth, Proc. Roy. Soc. London 273, 247 (1963).

${ }^{22}$ T. Kanomata, H. Ishigaki, T. Suzuki, H. Yoshida, S. Abe, and T. Kaneko, J. Magn. Magn. Mater. 140-144, 131 (1995).

${ }^{23}$ R. Lizarraga, L. Nordstrom, L. Bergqvist, A. Bergman, E. Sjostedt, P. Mohn, and O. Eriksson, Phys. Rev. Lett. 93, 107205 (2004). 